\title{
Electro-optical dust sensors calibration by aerosol image analysis
}

\author{
Vladimir Semenov ${ }^{1, *}$, Sergey Makov ${ }^{1}$, Yurij Hanzhonkov ${ }^{1}$, and Yurij Astsaturov ${ }^{2}$ \\ ${ }^{1}$ Don State Technical University, Department of Radio-electronic and electro-technical systems, \\ 147 Shevchenko st., 346500 Shakhty, Russia \\ ${ }^{2}$ Don State Technical University, Department of Technical operation of cars, 147 Shevchenko st., \\ 346500 Shakhty, Russia
}

\begin{abstract}
The paper describes the process of data obtaining for designing electro-optical sensors using a television aerosol particles image analyzer. Using simulation based on Mie theory, the process of calibration of simple electro-optical aerosol (dust) sensor is carried out. In the paper the main dependences for sensor calibration are shown. The proper calibration allows power saving by selection certain laser beam power level.
\end{abstract}

\section{Introduction}

In the development of optical-electronic sensors to control the concentration of industrial dust, there is the problem of their graduation for a specific type of dust. In some cases, it is necessary to calibrate the sensors only on the site of their installation or in laboratory conditions on special stands.

At the same time, the currently existing mathematical models allow to design the parameters of such sensors with sufficient accuracy for practical implementation $[1,2,5]$. However, some of the required input data in computer simulation algorithms is difficult to obtain without experiments. Existing television aerosol analyzers helps to obtain he missing data to make adequate models of electro-optical sensors.

Obtaining adequate model of dust allows use optimal conditions for electro-optical sensors. It means that we can set the optimal level of laser beam power. And finally it leads to common power saving in this sphere.

\section{The model}

To obtain input data for computer modeling, it is proposed to use television measuring devices, whose work is based on image analysis of particles of industrial aerosol flowing through a measuring chamber $[3,4]$. These devices allow obtaining and storing in the computer database the basic parameters of the aerosol. Those parameters are needed for modeling, calculating and calibrating of electro-optical sensors. Using this approach you can quickly reconfigure existing sensors for a specific type of industrial dust. This allows

\footnotetext{
*Corresponding author: semenov@sssu.ru
} 
attenuation the development time of electro-optical sensors and expanding the range of their application. A television measuring device forms a database characterizing the shape and optical parameters of industrial aerosols, and can be used as a reference tool for the calibration of electro-optical sensors based on the attenuation and scattering of optical radiation by industrial dust.

Television aerosol analyzer allows obtaining the following parameters for modeling:

- the aerosol belonging to spherical or non-spherical particles (the choice of an appropriate computer model based on calculation methods depends on: the method of separation of variables, the method of perturbations or direct methods based on the exact solution of the Maxwell integral equation);

- average particles size $\delta$, in micrometers;

- normalized distribution function of aerosol sizes;

- aerosol density $\rho$, in $g / \mathrm{m}^{3}$ obtained from analysis of color, texture of aerosol particles image;

The input data for modeling program are:

- complex value of refractive index $(\bar{n}=n+i x)$;

- average particles size $\delta$, in micrometers;

- wavelength $\lambda$, in micrometers;

- normalized distribution function of aerosol sizes;

- aerosol density $\rho$, in $\mathrm{g} / \mathrm{m}^{3}$;

- dissipation angle quantization step $\Delta \Theta$,in radians;

- initial and final angle value $\Theta$, in radians;

The output data of the modeling program are:

- values of series coefficient $A_{m}$ used for mathematical model stability analysis for range of input data;

- some of intermediate values of computing such as $a_{m}, b_{m}, K_{0}(x), K_{p}(x), S_{1}(x)$, $S_{2}(x), m_{1}(\Theta, x), m_{2}(\Theta, x)$ for the further analysis;

- the weight coefficient of radiation dissipation $\beta_{p s}, \mathrm{~m}^{2} / \mathrm{g}$;

- the weight coefficient of radiation attenuation $\beta_{o s}, \mathrm{~m}^{2} / \mathrm{g}$;

- normalized indicatrix value of radiation dissipation $P(\Theta)$.

With the help of a simulation program, coefficients characterizing the optical properties of a controlled industrial aerosol can be calculated.

With using the expressions obtained in [3] the calibration function of electro-optical sensors based on dissipation is following:

$$
U_{P}=K_{I P} \beta_{P B} P(\Theta) C \cdot e^{-\beta_{O B} L C}
$$

here $L=l_{1}+l_{2}$ - the distance traveled by the beam in the dusty aerosol from the exit lens of the illuminator to the entrance lens of the photo-detector.

For electro-optical sensor the dependence of output signal $U_{0}$ on radiation attenuation and aerosol concentration is following:

$$
U_{0}=K_{0}\left[1-\left(1+L \beta_{P B} P(\Theta) w_{2} C\right) \cdot e^{-\beta_{O B} L C}\right]
$$

These dependences were studied within real electro-optical sensors. An algorithm, a block diagram and a program for calculating the parameters of electro-optical sensors of 
industrial dust, based on the dissipation and attenuation of radiation, have been developed. The input data for the program are the following parameters:

- the weight coefficient of radiation dissipation $\beta_{p 8}, \mathrm{~m}^{2} / \mathrm{g}$;

- the weight coefficient of radiation attenuation $\beta_{o s}, \mathrm{~m}^{2} / \mathrm{g}$;

- normalized indicatrix value of radiation dissipation $P(\Theta)$ for the certain particles size;

- distance traveled by beam in aerosol from exit lens of the illuminator to the control point $l_{1}$ in meters;

- distance traveled by beam in aerosol from the control point to then input lens of the entrance lens of detector $l_{2}$ in meters;

- solid angle of spreading dissipated radiation in surface of detector $w_{2}$ in radians;

- gain coefficient $K$;

- detector sensitivity $S$;

- optical attenuation of lenses system $\tau$;

- power of beam $\Phi_{0}$.

The output data of the program is the dependence of sensor voltage (current) on aerosol concentration $C$. The obtained dependences allows determinating of optimal tuning of electro-optical sensors.

To obtain the model's input data, it is proposed to use a television measuring device, whose operation is based on television analysis of images of industrial aerosol particles flowing through a neasuremet chamber volume, which allows determining and storing in the computer the main aerosol parameters necessary for modeling, calculating and calibrating electro-optical sensors.

Picture 1 and 2 shows examples of calibration dependences obtained with mentoned expressions (1) and (2). Showed graphics are for the aerosol of coul dust with particles sixa les than 30 micrometers. The first graphic shows increasing the light power due to scattering light in aerosol.

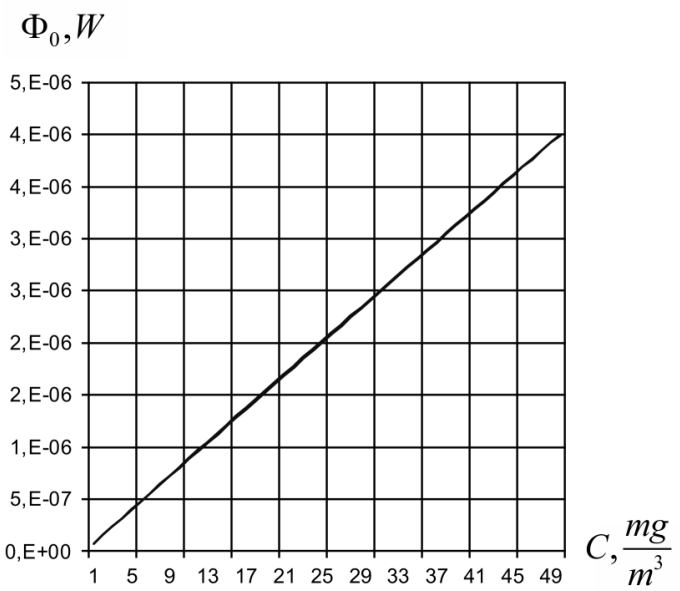

Fig. 1. Dependence of light scattering on aerosol concentration.

The second graphic shows decreasing the light power due to attenuation of direct light into aerosol with growing concentration. 


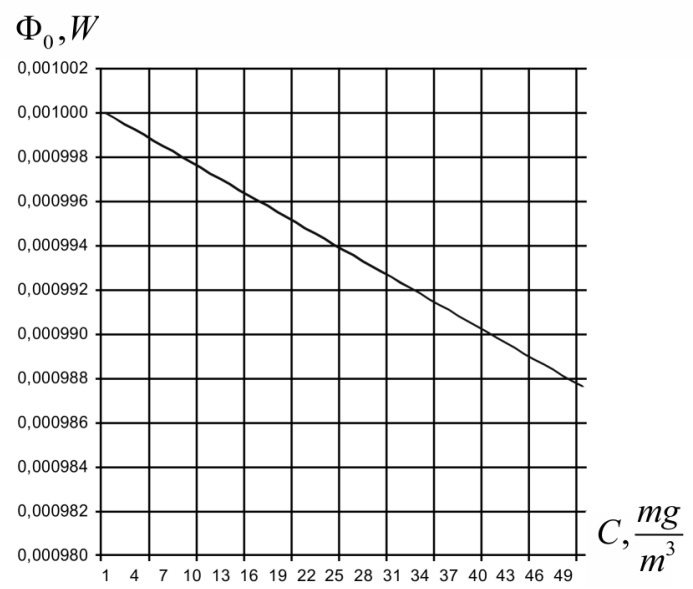

Fig. 2. Dependence of light power on aerosol concentration.

\section{Conclusion}

The compiled algorithms and programs allow modeling the processes of attenuation and light scattering by particles of industrial aerosol and the operation of electro-optical sensors of industrial dust based on these processes, which allows to automate the process of their development. Also it helps to select optimal parameters of electro-optical sensors, particular the laser beam power level to save energy.

Using this approach it is possible quickly reconfigure existing sensors for a specific type of industrial dust. This allows to reduce the development time of electro-optical sensors and expand the range of their application.

Currently, the authors are developing computer models for non-spherical of aerosol particles. The obtained result can be used in sphere of evaluation of electronic cigarette aerosol particle size [6].

\section{References}

1. C. Bohern, D. Huffman, Absorption and scattering of light by small particles (A Wiley-Interpscience Publication Jhon Wiley \& sons, 1983)

2. D. Deirmendjian, Electromagnetic scattering on spherical polydispersions (Elsevier, New York 1969)

3. V. Semenov, Izvestiya SFedU. Engineering sciences, 10 (2014)

4. V. Semenov, Y. Khanzhonkov, Y. Astsaturov, Engineering Journal of Don, 4 (2012)

5. P. Kulkarni, P. A. Baron, K. Willeke (Eds.). Aerosol measurement: principles, techniques, and applications (Wiley, New York, 2001).

6. E. L. Floyd, L. Queimado, J. Wang, J. L. Regens, D. L. Johnson, PloS one, 13, 12, e0210147 (2018) 